\title{
Compacidad Urbana: Estrategia Metodológica en pro de la Complejidad de las Ciudades
}

\author{
Urban Compactness: Methodological Strategy in Favor of the Complexity of \\ Cities
}

Mariaelisa Quintero Bosetti ${ }^{1}$

Recibido: 07 de septiembre de 2016

Aceptado: 26 de diciembre de 2016

\section{Resumen}

Los problemas en la ciudad registrados desde finales del siglo pasado, como su densificación, dispersión y constantes cambios en la morfología, han generado conflictos de toda índole, para los cuales las ciudades no están preparadas. Estos problemas han afectado directamente al ecosistema urbano e incluso su permanencia. Estudios recientes acerca de ecología urbana promueven el aumento de la complejidad de las ciudades, dando prioridad al ejercicio de la ciudadanía, entendida como las relaciones de contacto, intercambio y especialmente comunicación, esencia indiscutible de la ciudad. El objetivo principal de este artículo fue proponer un modelo de análisis operacional destinado a establecer criterios de diseño dirigidos a la consolidación de ciudades compactas, sostenibles en lo social, económico y ambiental, que propendan a la cohesión urbana. Se trata de un esquema metodológico cuyos atributos e indicadores no transgreden normativas generales de ordenación urbana o ambiental vigentes, sino que es susceptible de integrarse a planes macro de ordenación urbanística, local, especial y ambiental y puede ser aplicado a zonas o sectores de ciudades latinoamericanas que ofrezcan la interacción de multifocalidades que, al ser tratadas adecuadamente, pueden conducir a la resiliencia de las ciudades.

Palabras clave: ciudades compactas, complejidad urbana, modelo de análisis.

\begin{abstract}
Problems registered at the end of the last century and in the beginning of the current century such as the urban density, urban sprawl and the constant changes in the morphology of the cities, have created conflicts of all kinds, for which, the cities were not prepared to confront. These problems have affected directly the urban ecosystem and even its permanence. Recent studies about Urban Ecology promote the increase of the complexity of the cities giving priority to the exercise of citizenship. That is to say, to encourage the relations of contact, exchange and the unquestionable essence of the city, the communication. With the purpose of following this tendency, the object of this research is to propose an Operational Analysis Model aimed to establish standards of design directed to the consolidation of compact cities, socially, economically and environmentally sustainable, which favors the urban cohesion. It is a methodological scheme whose attributes and indicators do not infringe these current general regulations of urban and environmental planning. But instead it is susceptible to integrate itself into macro plans of urban, local special and environmental planning and it could be applied to areas or sectors of Latin American cities that could offer the interaction of potentialities that, if they are treated properly, will be susceptible to help cities to become more resilient.
\end{abstract}

Keywords: analysis method, compact cities, urban complexity.

\footnotetext{
${ }^{1}$ Universidad de Los Andes, Facultad de Arquitectura y Diseño, Grupo de Investigación de Calidad Ambiental Urbana (GICAU), Mérida, Venezuela. Contacto: qmarielisa@hotmail.com
} 


\section{Introducción}

El proceso urbano de los últimos cincuenta años es la historia de hacer la ciudad desde la arquitectura, con base en el deseo de la humanización de la ciudad, es decir, pensando en el hombre. Una arquitectura con sentido humano, por y para el hombre, dejando siempre al medio ambiente como telón de fondo.

Desde la Segunda Guerra Mundial hasta los límites del siglo XXI, se aprecia una gran cantidad de fenómenos urbanos sin precedentes. La extraordinaria expansión de las urbes es consecuencia no solo de la reconstrucción de algunas ciudades después de la guerra, sino sobre todo del éxodo de los habitantes de las zonas rurales a las ciudades con actividades industriales y mayor estabilidad económica.

Por ello, la organización espacial de dichos procesos sociales y estructurales producidos por el comportamiento del hombre, hacen que la ciudad se comporte de modo disperso, y que la continuidad de la misma sea proporcionada infraestructuras, no por las relaciones humanas de contacto, regulación, intercambio y comunicación propias del ecosistema llamado ciudad. Para Salvador Rueda (1995) queda claro que los propósitos que guían la construcción de la ciudad actual no tienen como prioridad el aumento de la complejidad de la ciudad, es decir, aumentar la probabilidad de contactos, intercambios y comunicación (que es, en definitiva, la esencia de la ciudad y la que tendría que guiar su construcción).

Las ciudades sufrieron cambios importantes en su morfología luego de la generalización del uso del automóvil y tuvieron que adaptarse: se introdujeron nuevas calles y se colocaron las primeras señales de tránsito. La mayoría de las ciudades tradicionales no se encontraba preparada para dichos cambios, presentando problemas de congestión (atascos frecuentes), así como también la homogenización de los espacios, usos no compatibles y consumos de enormes cantidades de energía para la movilidad, aspectos propios de las llamadas ciudades difusas.

Se puede afirmar que el crecimiento de las nuevas áreas urbanas va de la mano de la red de movilidad. Para resolver los conflictos que se generan a partir de esto, se hace necesaria la ocupación de mayores espacios (generalmente sin respetar áreas naturales ni intersticios urbanos), aumentando la presión sobre la explotación desmedida de los recursos que la alimentan.

En este sentido, por muchos años se ha pensado que el proceso de urbanización es un fenómeno demasiado rápido y desordenado. Actualmente, la observación del sistema de ciudades es utilizada para la identificación y jerarquización de los asentamientos urbanos como base para la distribución 
equilibrada de las dotaciones, sobre todo, de los equipamientos básicos públicos y privados (educativos, culturales, sanitarios, deportivos y asistenciales) (Banco Mundial, 2010).

Según Girardet (1992), dicha jerarquización responde a criterios de peso demográfico, cantidad y especialización de las funciones urbanas y capacidad de atracción en términos de movilidad y accesibilidad, en relación con su influencia y radios de acción respecto de otros núcleos urbanos. De la misma manera, esta tendencia a diluir la complejidad de las ciudades es afrontada por el autor cuando afirma que la ciudad como una matriz compleja y cambiante, debe constituirse por un "metabolismo circular", en el que la reutilización de recursos reduzca el impacto de la huella ecológica.

Esta idea de diversidad y cohesión social, donde todas las actividades humanas se entrelazan y reavivan el sentido de pertenencia en todos sus habitantes se fundamenta en el concepto de ciudades compactas. Algunos autores concuerdan en que la respuesta para lograr la sostenibilidad de nuestras ciudades, además de reducir los impactos ambientales es, sin duda, aumentar los niveles de complejidad urbana: intercambio, mezcla de etnias, densificación, diversidad de usos y lograr la revitalización urbana.

Dicha complejidad incentiva el contacto y el intercambio necesarios para la vida en sociedad y, para lograr la revitalización urbana, es tácita la idea de que la movilidad y accesibilidad son fundamentales al pensar en la ciudad, por tanto, la mezcla de equipamientos y el aumento de los espacios públicos provee de una mayor sensación de seguridad social, disminuye los desplazamientos $y$, por ende, el consumo mínimo de territorio para cubrir dichas necesidades.

Una vez esbozadas las características de las ciudades difusas y compactas es importante hacer un apartado respecto del inusual proceso de urbanización de las ciudades latinoamericanas en comparación con el de otras ciudades del mundo, ya que fueron planificadas como parte de un proceso de conquista, brindándoles características especiales que solo fueron superadas siglos después con la llegada del pensamiento moderno.

Conocedores de la materia como Amaya (2011), Pérgolis (2005) y Pesci (2002) y coinciden en que este proceso urbano se resume en tres tiempos del urbanismo latinoamericano:

- La ciudad continua, con asentamientos coloniales que permanecieron en algunos casos hasta la década de 1950.

- La ciudad discontinua (en consonancia con el pensamiento moderno), con la especialización de usos y la reorganización de la morfología de la ciudad.

- La ciudad fragmentada o difusa, coherente con la globalización y el modo caótico de vida contemporánea, donde impera el individualismo como reemplazo de los modelos ideológicos, abundan cambios de uso de suelo, 
disminución del espacio público y la degradación general del medio ambiente urbano.

Es importante resaltar que, sin embargo, en este panorama existen notables excepciones y una clara propensión a generar nuevas políticas urbanoambientales, producto de la toma de conciencia del valor del patrimonio histórico, social, arquitectónico y urbanístico de nuestras ciudades.

Consecuentemente, si se considera que la forma de las ciudades es determinante en el modus vivendi de sus habitantes, es evidente que la organización del territorio y la red de movilidad son parte importante de ello, ya que definen la fragmentación de espacios y usos, precisando entonces, las distancias y los niveles de consumo de energía necesaria para cubrirlas, es decir, la construcción de más vías, generación de nuevos espacios residuales y mayor dependencia del transporte motorizado. Estas complejas condiciones de funcionamiento generan, además, mayores niveles de contaminación, polución e insostenibilidad ambiental y energética. Asimismo, la inclusión de la dimensión ecológica en el quehacer urbano, en cuanto a la moderación o la resolución de la crisis ambiental a la que se enfrentan nuestras ciudades, se sustenta en el hecho de que estas representan la forma más concreta de interacción entre la sociedad y el entorno natural.

Estos paradigmas se basan en postulados dirigidos a la consolidación de ciudades sostenibles, social, económica y ambientalmente, a fin de minimizar y evitar las patologías que padecemos en nuestras urbes, tales como el deterioro en los servicios y equipamientos urbanos, congestión vehicular, segregación de usos y una clara disminución en la calidad ambiental y espacios públicos, entre otros.

De allí que el presente artículo se inicie con el cotejo de postulados y proyectos de enfoques ecológicos y sistémicos (sus restricciones y potencialidades), a fin de que sirvan de marco epistemológico para la propuesta de un modelo de análisis operacional que incorpore lineamientos tendientes a regular las intervenciones en ámbitos urbanos y presente la oportunidad de responder a la hipótesis de si una ciudad compacta es una ciudad sostenible.

\section{Marco teórico}

Lograr la sostenibilidad ambiental en Latinoamérica representa un gran reto, pues la rápida expansión urbana y sus consecuencias económicas, sociales y medioambientales, se ven expresadas en las tendencias de los indicadores que señalan una grave degradación de los recursos naturales, mermas en la productividad y el ingreso, vulnerabilidad física y deterioro de la calidad de vida. 
Debe destacarse que las ciudades intermedias de la región (de tamaño demográfico de cincuenta mil a un millón de habitantes) cuentan con condiciones propias que les imprimen mayores probabilidades de ser redireccionadas, para recuperar su compacidad original y mejorar sus condiciones urbano-ambientales, con miras a lograr la sostenibilidad. Se trata de ciudades que por sus ubicación geográfica, tamaño demográfico y creciente desarrollo económico, tienen mayores probabilidades de dirigir sus esfuerzos hacia la descentralización y, por lo tanto, trabajar desde las bases municipales en el mejoramiento de los procesos de gestión urbana en sus respectivos contextos, fungiendo como verdaderos promotores del desarrollo local y no como simples administradores locales de los recursos del gobierno central.

Desde esta perspectiva, es evidente que el establecimiento de estas metas e indicadores son una reafirmación de los propósitos planteados en el año 2000 en la Cumbre del Milenio en la ciudad de Nueva York, en donde se impone la importancia de que exista un principio común entre la política, la planificación, la sociología y los demás sectores de la sociedad, a fin de establecer fundamentos adecuados para lograr una mayor eficiencia en la formulación y aplicación de las nuevas estrategias públicas y en la búsqueda de la sostenibilidad de las ciudades.

Dispersión versus compacidad. Como se ha mencionado hasta ahora, el crecimiento de las ciudades admitió absorber pueblos y ciudades vecinas, formando conurbaciones urbanas compuestas de varios ámbitos suburbanos que se diferencian funcional y orgánicamente, y donde cada uno de ellos presenta una organización propia del espacio. Desde el punto de vista espacial, y para mantener la continuidad, en el modelo de ciudad difusa es necesario hacer uso de sistemas complejos de carreteras que acerquen funcionalmente dichos ámbitos suburbanos, creándose vacíos internos en la ciudad y la inevitable pérdida de contenido e identidad.

Existe consenso a escala mundial respecto de que el proceso de urbanización es un fenómeno veloz y casi inmanejable. Por tal razón, hoy en día se recurre a la observación del sistema de ciudades con el fin de identificar y jerarquizar los asentamientos urbanos como base para la distribución equilibrada de las dotaciones, sobre todo de los equipamientos básicos públicos y privados (educativos, culturales, sanitarios, deportivos y asistenciales). Dicha jerarquización según Girardet (1992), responde a criterios de peso demográfico, cantidad y especialización de las funciones urbanas y capacidad de atracción en términos económicos, de movilidad y accesibilidad, en relación con su influencia y radios de acción respecto de otros núcleos urbanos.

La compacidad como modelo urbano. El concepto de ciudad compacta en Europa surge como alternativa ante la masiva suburbanización 
producto de la segunda Guerra Mundial, así como las ciudades jardín fueron la reacción a las ciudades súper pobladas de siglo XIX (Breheny, 1996 citado por Westerink et al., 2013).

La aplicación del modelo de ciudad compacta se fundamenta originalmente en la proximidad, pero posteriormente ha sido sustituido por el término de accesibilidad, dejando espacio a las multifocalidades (Westerink et al., 2013), en las que todas las actividades humanas se solapan y se reaviva el sentido de pertenencia en todos sus habitantes. Algunos autores concuerdan en que la clave para lograr la sostenibilidad de nuestras ciudades, radica en la reducción de la necesidad de movilización (número de viajes), la consolidación del transporte público como medio principal y en el uso eficiente de los espacios urbanos con el propósito de reducir los impactos ambientales; por otra parte, aumentar los niveles de complejidad urbana, expresados en términos de densificación, diversidad de usos, mezcla de etnias, aumento de la red asociativa-ámbito social y, finalmente, lograr la revitalización urbana (Echenique, Hargreaves \& Mitchell, 2013).

La morfología de las ciudades es determinante en el comportamiento de sus habitantes, por ello es que la organización del territorio y la red de movilidad son parte fundamental. El hecho de entender a la ciudad como un sistema complejo compuesto por muchos subsistemas, requiere de un eficiente manejo de cada uno de ellos, lo que involucra factores físico-espaciales, socioeconómicos y de gestión urbana (Carmona, 2010). Actualmente la globalización encamina a las ciudades a fortalecer su competitividad, aprovechando las ventajas de su ubicación, tamaño, accesibilidad, servicios y la capacidad de dar respuesta ante las crecientes demandas de nuevos espacios urbanos (Chavoya, García y Rendón, 2009). De acuerdo con esto, Westerink et al. (2013) afirma que los niveles de densidad (medida cuantitativa) y/o intensidad (medida cualitativa) del uso de suelo, son indicadores de urbanidad, por lo que mientras más densa sea una ciudad, más urbana es la misma, característica fundamental de las ciudades compactas.

Es importante señalar que actualmente existen posturas o líneas argumentativas contrarias al ideal propuesto por la compacidad urbana, sustentadas en la incertidumbre de cuantificar si este modelo en sí es realmente sostenible y/o si las metas alcanzados tras su aplicación compensan posibles impactos "negativos" en el medio ambiente y en la calidad de vida de sus habitantes. Así, por ejemplo, para Echenique et al. (2013) y Gaigné (2012) los logros medio ambientales obtenidos (reducción de las emisiones de $\mathrm{CO}_{2}$, eficiencia en el manejo de las energías y de los espacios urbanos, entre otros) son relativamente pequeños en relación con las afectaciones en lo socioeconómico, en virtud de que las políticas de densificación son ambiguas en cuanto a la ocupación real del territorio, pudiendo generar más polución y alguna disminución en el confort de las viviendas. Estudios recientes realizados en ciudades tanto europeas como norteamericanas demuestran que dichas 
implicaciones pueden ser opacadas o disminuidas por el acceso a la vitalidad urbana (relación tamaño de la vivienda versus ubicación, servicios, espacios públicos y equipamiento).

De todas forma, el concepto de ciudad compacta admite varias interpretaciones y posibilidades de implementación, ya que requiere del análisis de las cualidades específicas de cada región, es decir, el estudio de su contexto histórico, etnográfico, físico, económico y ambiental (Salingaros, 2007). Como se expresa en la Tabla 1, a modo de resumen y con el propósito de dejar claramente establecidas las características de los modelos de ciudades compactas y difusas, se presenta un cuadro comparativo que considera atributos válidos para este artículo.

Tabla 1

Dispersión versus compacidad

\begin{tabular}{|c|c|c|}
\hline Características & Ciudad difusa & Ciudad compacta \\
\hline $\begin{array}{c}\text { Morfología } \\
\text { urbana }\end{array}$ & $\begin{array}{l}\text { - Dispersa, mayor ocupación } \\
\text { de territorio con menor } \\
\text { densidad de población } \\
\text { (menos hab./ hectárea) } \\
\text { - Menor cohesión social. }\end{array}$ & $\begin{array}{l}\text { - Compacta, menor } \\
\text { ocupación de espacio } \\
\text { físico y mayor densidad } \\
\text { poblacional. } \\
\text { Mayor intercambio y } \\
\text { diversidad social. }\end{array}$ \\
\hline Movilidad & 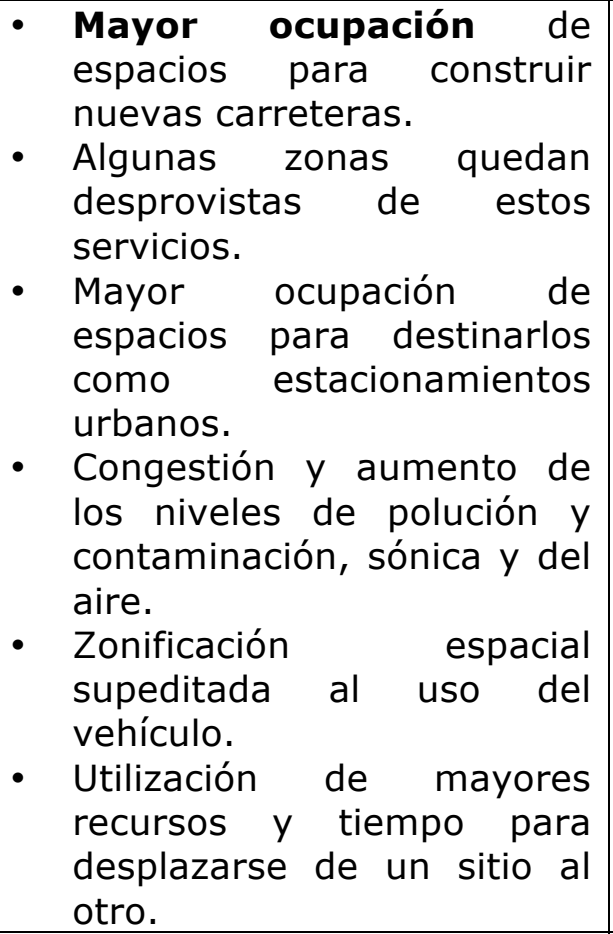 & $\begin{array}{l}\text { - Menor ocupación de } \\
\text { espacios urbanos para la } \\
\text { movilidad rodada. } \\
\text { - Distribución igualitaria } \\
\text { del servicio. } \\
\text { - Menor congestión y } \\
\text { reducción en los niveles } \\
\text { de contaminación } \\
\text { ambiental. } \\
\text { - Reducción del consumo } \\
\text { de recursos. } \\
\text { Menores distancias entre } \\
\text { servicios } \\
\text { equipamientos, lo que } \\
\text { favorece la movilidad } \\
\text { peatonal y sostenible. }\end{array}$ \\
\hline Usos de suelo & $\begin{array}{l}\text { - Zonificación y } \\
\text { especialización de las zonas } \\
\text { urbanas. }\end{array}$ & 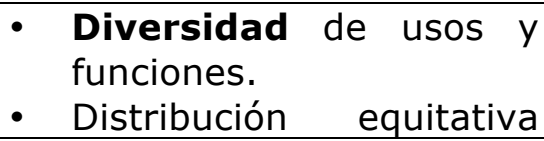 \\
\hline
\end{tabular}




\begin{tabular}{|c|c|c|}
\hline & $\begin{array}{l}\text { - Beneficio de unas áreas } \\
\text { sobre otras. (equipamientos } \\
\text { desiguales). }\end{array}$ & $\begin{array}{l}\text { de servicios } \\
\text { equipamientos. } \\
\text { - } \quad \text { Igualdad social. } \\
\end{array}$ \\
\hline $\begin{array}{l}\text { Espacio } \\
\text { público }\end{array}$ & $\begin{array}{l}\text { - Zonas reducidas a } \\
\text { espacios aislados y de } \\
\text { poco valor para el } \\
\text { ciudadano común. } \\
\text { - Aumento de la inseguridad } \\
\text { y menor vitalidad social. } \\
\text { - Accesibilidad condicionada, } \\
\text { inexistencia de redes } \\
\text { peatonales continuas y } \\
\text { claras. } \\
\text { Menor oferta de zonas } \\
\text { verdes. }\end{array}$ & 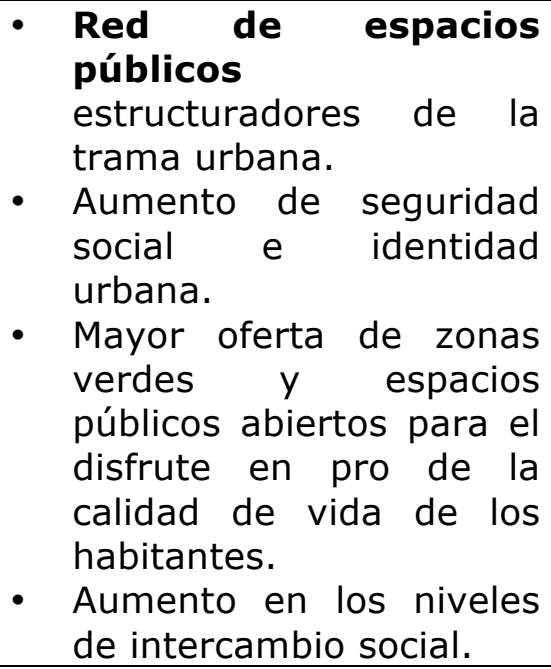 \\
\hline $\begin{array}{c}\text { Metabolismo } \\
\text { urbano }\end{array}$ & $\begin{array}{l}\text { - Lineal: consumo } \\
\text { despreocupado de los } \\
\text { recursos del medio } \\
\text { ambiente (Girardet, 1992). } \\
\text { - Superación de los límites de } \\
\text { carga, sin pensar en planes } \\
\text { de reciclaje y utilización de } \\
\text { los mismos. }\end{array}$ & 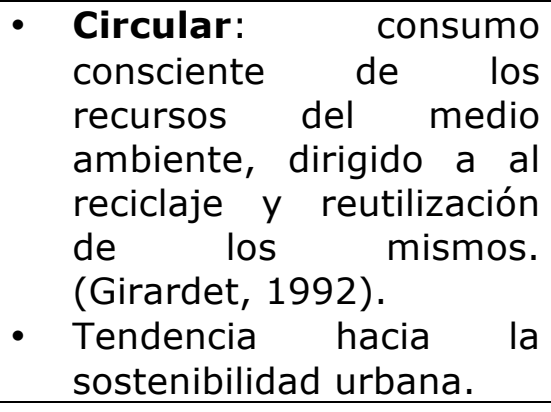 \\
\hline
\end{tabular}

Fuente: Quintero B. (2012).

Revisados algunos de los avances y conflictos de las ciudades anteriormente expuestos, se procede a desarrollar algunas de las teorías y métodos que han surgido específicamente en el campo del urbanismo y que, entre otros, son primordiales para esta investigación:

- Reservas de Biosfera en Ambientes Urbanos (RBAU), en tanto que adaptación del modelo metodológico de Reserva de Biosfera Natural.

- Nuevo Urbanismo de Duany y Plater-Zyberk (2003), que plantea la intervención urbana a través de ordenanzas disponibles para todas las escalas de la planificación y se fundamenta en el estudio de la relación medio ambiente-entorno construido,

- Teoría de la morfogénesis (Ecoforma y Socioforma) de Rubén Pesci (2004).

A continuación se revisarán estos aspectos. 
Reservas de Biósfera Urbanas (RBU). Partiendo de la idea de que la ciudad es un ecosistema, Pesci (2004), siguiendo el esquema metodológico del concepto de Reservas de Biosfera Naturales (Unesco, 2011) dirigieron su atención al estudio de los ecosistemas urbanos, clasificando el espacio urbano y su transición con el medio ambiente en cinco zonas de intervención; este planteamiento depende de la participación y apoyo de todos los actores involucrados.

La clasificación de las zonas de intervención es la siguiente:

- Zona de núcleo urbano: correspondiente a las zonas urbanas consolidadas que ostentan patrimonio natural y construido.

- Zona de amortiguación urbana: referente a la interfase entre la amortiguación urbana y la natural.

- Zona de transición urbano-natural: caracterizada por la baja densificación y ocupación del territorio.

- Zona de amortiguación natural: es aquella inmediata al núcleo natural y en la que es factible realizar la función de conservación ambiental.

- Zona de núcleo natural: constituida por ecosistemas naturales representativos.

Nuevo Urbanismo. Corriente de planificación y diseño urbano que nació como respuesta ante las políticas urbanas del movimiento moderno. Sus principales representantes son los arquitectos estadounidenses Andrés Duany y Elizabeth Plater-Zyberk, quienes buscaron retornar a los métodos urbanísticos tradicionales americanos, donde el tejido urbano estaba basado en la variedad y mezcla de usos (Duany \& Plater-Zyberk, 2003).

Este movimiento apoya la idea de que la configuración urbana puede incentivar la cohesión social a través del equilibrio entre el hombre, sus actividades y su entorno, desarrollando el sentido comunitario de pertenencia.

Entre los principios que rigen el Nuevo Urbanismo se encuentran los siguientes:

- Tránsito peatonal y conectividad: a través de un diseño urbano orientado a estimular la movilidad.

- Diversidad y mixtura de usos.

- Aumento de la densidad y complejidad urbana.

- Movilidad sostenible impulsada por sistemas de transporte público.

- Sostenibilidad ambiental.

- Arquitectura de calidad a fin de recuperar el sentido de pertenencia y lograr escalas más humanas. 
De esta experiencia resulta Seaside (Florida, EEUU), proyecto piloto en la década de los 80 como "una alternativa de ciudad que piensa en el hombre, en el ambiente natural y en la vida en comunidad; sin dejar de lado la importancia de las actividades económicas, culturales, recreativas y reglamentarias que la sostienen" (Remar, 2004). El mérito de esta intervención radica en que a partir de ella se elaboraron códigos diferentes para otros lugares que incluyen en sus planteamientos un mayor compromiso medio-ambiental.

Dos décadas después de la elaboración de este documento, en el año 2003, los arquitectos creadores de la teoría del Nuevo Urbanismo desarrollaron y presentaron un instrumento de planificación y diseño urbano llamado SmartCode que, con un enfoque ambientalista y pragmático de la forma urbana, buscó revertir las consecuencias medioambientales que generaron las ciudades difusas y la planificación expansionista basada en el pensamiento moderno.

Duany y Platter-Zyberk (2003) basados en la teoría de la transección (que permite clasificar el tipo de crecimiento y desarrollo de un espacio) y aplicando métodos de protección ambiental, proponen el corte transversal como un método eficaz para la descripción, identificación y formulación de zonas transecto en un área determinada, cuya gradualidad propuesta va desde los entornos más naturales a los más urbanos.

Morfogénesis. Modelo formulado por el arquitecto argentino Rubén Pesci (2004) a través de la Fundación CEPA (Centros de Estudios y Proyectación del Ambiente). Esta propuesta teórica nace a partir del concepto de interfases o zonas de encuentro y de la superposición de sistemas, a fin de entender la complejidad de las ciudades y establecer cuál es la organización espacial de las actividades y necesidades de un área urbana específica.

El autor afirma que la definición de morfogénesis se refiere a cómo dar forma a un proyecto, más que generar información es la manera de conceptualizar la realidad, se trata entonces de pasar de la información a la ideación, al dar forma concreta a las ideas, convirtiéndolas en iniciativas y proyectos fácticos. De igual modo explica que este modelo determina dos dimensiones objeto de estudio: las ecoformas y socioformas, con una visión de sostenibilidad subyacente.

La ecoforma. "Es la forma que consigue develar los ecosistemas ya sean dominantemente naturales o culturales, para conservarlos, manejarlos bien y acentuar su naturaleza, la que surge de su naturaleza, de su propia identidad" Pesci (2004, p. 28) y puede ser estudiada e intervenida en tres escalas: la territorial (planificación), la urbana (diseño) y la edilicia (arquitectura).

De igual manera el autor la define a partir de tres aspectos: 
a) El sistema de unidades naturales a proteger que depende de la valoración del entorno inmediato de las ciudades.

b) Los flujos de materia y energía, se corresponden con la idea de metabolismo urbano circular y principios ecosistémicos de renovación energética y de recursos.

c) El hábitat sustentable, referente a la compacidad de las ciudades, la ocupación del suelo y su relación con las tipologías arquitectónicas (Pesci, 2004).

La socioforma. "Es el contenido social y cultural del habitar el ambiente (su utilización), su adecuado manejo social y productivo que puede adquirir múltiples formas, no solo funcionales sino metafóricas" (Pesci, 2004, p. 28).

Esta dimensión es definida por el autor a partir de cinco aspectos:

a) La multipolaridad, concibe la ciudad como múltiples núcleos de vida interrelacionados.

b) La urbanidad y los espacios abiertos, fomentan la generación de espacios públicos dentro de la trama urbana para reforzar la idea de civis o urbanidad.

c) Los flujos de información, referidos a las comunicaciones en general, (social, cultural, política, económica y tecnológica, entre otras), que propician la toma de decisiones acerca del ámbito urbano.

d) La participación social, donde tiene lugar la legitimidad de las decisiones en toda acción de transformación de la ciudad, a través de la participación directa e indirecta de los ciudadanos.

e) La producción concertada de la ciudad, la unión de los aspectos anteriores debe apuntar a la concertación de los medios de producción que hacen vida en la ciudad, articulando intereses y prioridades.

Como se ha podido observar, a partir de la revisión de patrones urbanos existentes, se pueden proponer diferentes vertientes en la puesta en práctica de planes y criterios en la concepción de ciudades: unos basados en tendencias funcionalistas (ciudades difusas) y otros en pro de la sostenibilidad urbana (ciudades compactas).

En este sentido, sobre la base de los aportes mundiales a favor de la ecología urbana, en la revisión de las características físico-espaciales de las ciudades, en los postulados de las teorías y métodos del diseño urbano ambiental, así como en los avances y conflictos de las ciudades contemporáneas, se propone un modelo de análisis operacional, que sirva de base para el establecimiento de estrategias y criterios de diseño que, aplicados a pequeña escala, propendan a la promoción de la compacidad y sostenibilidad de las mismas. 
Como se aprecia, los sistemas urbanos son ecosistemas complejos y adaptables, compuestos por flujos metabólicos (intercambio de materias y energías), morfología urbana (forma e infraestructura), dinámicas socioculturales y redes de gobierno (gobernabilidad y gestión urbana), todos en diversas escalas interrelacionados entre sí.

De acuerdo con lo señalado, es inevitable pensar en sostenibilidad como enfoque para la reflexión y actualización de las prácticas urbanas. No obstante, el logro de una solución factible, de gran importancia para la elaboración de este trabajo de investigación, dependió del establecimiento de la relación unívoca entre la teoría y la práctica.

Teniendo presente que la idea de compacidad urbana permite variadas interpretaciones e implementaciones, el modelo de análisis operacional propuesto considera la observación de cualidades y especificidades propias de la ciudad, agrupadas en los atributos que conciernen a la morfología, la cohesión, y la movilidad urbana así como los usos de suelo y los espacios públicos. Es importante señalar que atributos tales como el metabolismo urbano (intercambio y uso de materiales y energía), no fueron tomados en cuenta, porque sus implicaciones son tan amplias y polémicas que deben ser objeto de estudios posteriores.

Autores como Camagni, Gibelli y Rigamonti (2002) y Fumega, Niza y Ferrão (2014) afirman que la forma urbana influye en la manera en que los residentes perciben e interactúan con su contexto inmediato, así como en la eficiencia y competitividad de la movilidad urbana y en el uso del suelo. A este respecto, la morfología urbana es analizada en esta investigación a través de elementos subyacentes, absolutos y semánticos. Subyacentes, aquellos que no son perceptibles a primera vista y están relacionados con las condiciones físiconaturales de la ciudad. Absolutos, aquellos relacionados con las características que le confieren los rasgos formales y funcionales; y finalmente, los elementos semánticos, los que trascienden a la percepción de los espacios. La movilidad urbana, por su parte, es estudiada desde la perspectiva peatonal y vehicular (transporte público, accesibilidad y eficiencia).

En términos de cohesión urbana y usos del suelo, el análisis se centra en la idea de que este último debe ser utilizado eficientemente con base en la densificación-intensificación, ya que en todos los aspectos contribuyen a la sostenibilidad, no solamente por sus beneficios en el uso de los recursos (reducción de $\mathrm{CO}_{2}$ y transporte público), sino que además estimula el intercambio social, la creatividad y la innovación (Christiaanse, 2009; Echenique et al., 2013; Manoug, 2013). Consecuentemente los espacios públicos se observan como el ámbito del ejercicio de ciudadanía, por cuanto refuerzan la imagen de la comunidad, el sentido de pertenencia entre los residentes mejorando la calidad de vida y la biodiversidad de las ciudades. 
Definición de los ecotonos. Como se ha descrito anteriormente, los postulados expuestos hasta ahora coinciden en la visión ecosistémica de la ciudad, así como también en la preponderancia de la dimensión ambiental, dentro de la toma de decisiones a la hora de intervenir espacios urbanos.

Por ello, en este artículo los ecotonos urbanos concebidos como áreas de interfases donde diversos ecosistemas naturales se encuentran con la ciudad, como posibles articuladores de la complejidad del sistema urbano, tienen el propósito de articular el diseño urbano con los postulados de acercamiento a la naturaleza, como espacios de integración, convivencia y dialogo (Bartorila, 2009).

Partiendo de esta definición, el sector caso de estudio será sectorizado en seis ecotonos (Figura 1), clasificados en una escala de gradualidad que acota de lo más natural a lo más urbano.
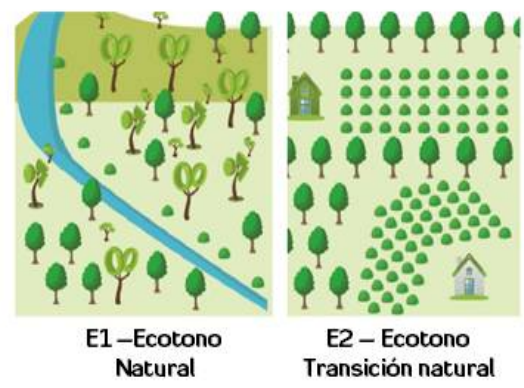

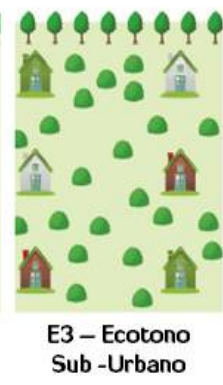

Sub -Urban
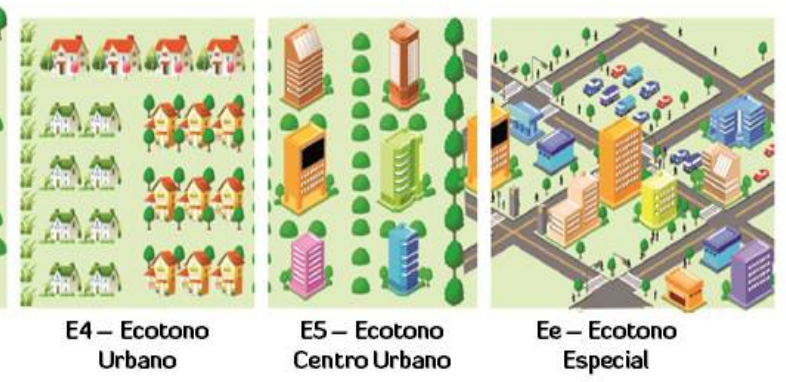

Fuente: Elaboración propia, 2012.

- E1-Ecotono Natural: compuesto por un ecosistema natural que incluye tierras no aptas para desarrollos urbanos debido a sus condiciones físiconaturales.

- E2-Ecotono Transición Natural: son zonas muy cercanas al ecotono natural con modelos de usos, cuya misión es la conservación de núcleo natural; incluye bosques y tierras con vocación agrícola.

- E3-Ecotono Suburbano: constituido por ámbitos cuyo propósito es el de articular los ecotonos agrícolas con los urbanos. Su característica principal es la baja densidad habitacional y de ocupación del suelo.

- E4-Ecotono Urbano: caracterizado por tejidos urbanos de usos mixtos y que requieren mejoras tanto de la calidad de vida como de la infraestructura, equipamientos e imagen urbana. Se trata de zonas en vías de consolidación.

- E5-Ecotono Centro Urbano: equivalente a zonas urbanas de alta densidad, consolidadas y con grandes posibilidades de lograr la sostenibilidad urbana. 
- Ee-Ecotono Especial: son áreas cuya función, disposición o configuración intrínseca no pueden conformar una de los cinco ecotonos antes descritos. Por ejemplo universidades, aeropuertos, industrias y fábricas.

Modelo de análisis operacional. Una vez expuestos los componentes conceptuales y definidos los ecotonos, se plantea un modelo de análisis operacional, que integra los enfoques mencionados anteriormente, y cuyo objeto es el de fundamentar estrategias de diseño urbano que promuevan la compacidad y la sostenibilidad de un sector urbano determinado.

De acuerdo con esto, y para hacer operativo este enfoque teórico-conceptual, se plantea el desarrollo de este modelo en tres grandes etapas consecutivas y complementarias: análisis del área de estudio, síntesis y aplicación del método.

Primera etapa: Análisis del área de estudio. Con la intención de una mayor comprensión del problema, esta etapa comprende una revisión exhaustiva de los elementos que configuran el sector, con el fin de priorizar las variables, indicadores e instrumentos a aplicar, para el análisis del caso de estudio.

Esta etapa se subdivide en tres momentos: diagnóstico conceptual, diagnóstico tipológico y síntesis.

- Diagnóstico conceptual: para caracterizar el caso de estudio a través de la zonificación en ecotonos del sector a intervenir.

- Diagnóstico tipológico: con el objetivo de estudiar los componentes urbanos del caso de estudio, es decir, los elementos subyacentes, absolutos y semánticos que conforman las variables que fundamentarán la posterior definición de los criterios de diseño de la propuesta urbana. Estas variables se estructuran en indicadores y se analizan a través de instrumentos. Es necesario precisar que en este trabajo los indicadores propuestos se fundamentan en algunos criterios tales como la sostenibilidad y la compacidad urbana, expuestos por varios autores y organismos internacionales (Rueda, 2007).

a) Análisis de los elementos subyacentes: concierne aquéllos elementos que no son perceptibles a primera vista, relacionados con las condiciones físico-naturales del sector. 


\begin{tabular}{|l|l|}
\hline \multirow{4}{*}{ Indicadores } & Ubicación y localización \\
\cline { 2 - 2 } & Topografía \\
\cline { 2 - 2 } & Clima \\
\cline { 2 - 2 } & Dirección de los vientos \\
\cline { 2 - 2 } & Vulnerabilidad \\
\hline \multirow{4}{*}{ Instrumentos } & Plano de ubicación y localización \\
\cline { 2 - 2 } & Plan topográfico y de vulnerabilidad \\
& \\
\hline
\end{tabular}

b) Análisis de los elementos absolutos: se refiere al estudio de las características que confieren los rasgos formales y funcionales al sector. Los indicadores aquí planteados siguen los lineamientos urbanísticos que promueven la sostenibilidad y la compacidad.

Es importante recordar que en la fundamentación teórica se establecieron los atributos que dan forma al modelo de ciudades compactas y, por ello, los indicadores se desarrollan siguiendo dichos atributos.

Así, siguiendo la lógica planteada, para el estudio del sector a intervenir se establece una Unidad de Análisis (UA) que se utiliza como área de referencia. En este caso se propone una medida estándar de $1.000 \mathrm{~m} \times 1.000 \mathrm{~m}$ (100ha). La elección de la dimensión de esta UA, responde al criterio del investigador y considera sectores con cualidades homogéneas que permiten delimitar el área objeto de estudio en zonas cuantificables y hace factible obtener resultados reales de la aplicación de los indicadores propuestos en la metodología.

Por esta razón, la UA es dividida posteriormente en cuatro sectores o microunidades de análisis de $500 \mathrm{~m} \times 500 \mathrm{~m}$, que constituyen una malla de referencia, a fin de acotar el área de estudio y circunscribirla a la escala de diseño urbano. Es importante señalar que esta subdivisión en microunidades ofrece la posibilidad de estudiar en detalle diferentes escenarios dentro de una misma unidad de análisis, y permite que los resultados obtenidos a partir de la aplicación de la metodología propuesta sean más precisos y cercanos a la realidad del área de estudio.

En la Tabla 2 se presenta el cuadro síntesis de los indicadores propuestos para el análisis de los elementos absolutos. 
Tabla 2

Sistema de indicadores

\begin{tabular}{|c|c|c|c|}
\hline \multicolumn{4}{|c|}{ Atributo: morfología urbana } \\
\hline Indicador & Objetivo & Resultados & Unidad \\
\hline $\begin{array}{l}\text { Caracterización } \\
\text { Espacial (CE). } \\
\text { (Forma urbana) }\end{array}$ & $\begin{array}{l}\text { Determinar el tipo de } \\
\text { trama o trazado urbano } \\
\text { desde su forma y } \\
\text { relación } \quad \text { con } \\
\text { espacios los } \\
\text { vacíos. llenos }\end{array}$ & $\begin{array}{l}\text { cE1= } \% \text { de } \\
\text { grano construido } \\
\text { y grano natural. } \\
\text { cE2= \% tipo de } \\
\text { trama. } \\
\text { cE3= hectáreas } \\
\text { de } \quad \text { espacios } \\
\text { abiertos. }\end{array}$ & $\begin{array}{c}\% \\
\text { Hectáreas }\end{array}$ \\
\hline $\begin{array}{l}\text { Densidad } \\
\text { edificatoria (De) }\end{array}$ & $\begin{array}{l}\text { Ordenar y regular el } \\
\text { crecimiento urbano. }\end{array}$ & $\begin{array}{l}\text { \% densidad real } \\
\text { de la unidad de } \\
\text { análisis. }\end{array}$ & $\begin{array}{l}\% \text { viv / ha } \\
\text { Valores }\end{array}$ \\
\hline \multicolumn{4}{|c|}{ Atributo: cohesión urbana } \\
\hline Indicador & Objetivo & Resultados & Unidad \\
\hline $\begin{array}{l}\text { Cohesión Absoluta } \\
\text { (Ca) }\end{array}$ & $\begin{array}{l}\text { Relacionar el volumen } \\
\text { edificado con el área de } \\
\text { la unidad de análisis } \\
\text { tomando en cuenta } \\
\text { únicamente la } \\
\text { intensidad edificatoria. } \\
\text { Este indicador } \\
\text { establece la altura } \\
\text { media de la edificación } \\
\text { de un área dada. }\end{array}$ & $\begin{array}{l}\text { Altura media de } \\
\text { las edificaciones. }\end{array}$ & $\begin{array}{c}\text { Altura } \\
\text { media de la } \\
\text { edificación/ } \\
\text { ha. }\end{array}$ \\
\hline $\begin{array}{l}\text { Cohesión Funcional } \\
\text { (Cf) }\end{array}$ & $\begin{array}{l}\text { Facilitar la accesibilidad } \\
\text { desde la vivienda a las } \\
\text { distintas actividades } \\
\text { habituales. }\end{array}$ & $\begin{array}{lr}\text { Valor } & \text { de } \\
\text { cohesión } & \\
\text { funcional /Plano } \\
\text { de radio de } \\
\text { acción } & \text { de } \\
\text { actividades } \\
\text { según distancias } \\
\text { recorridas. }\end{array}$ & Min/Metros \\
\hline \multicolumn{4}{|c|}{ Atributo: usos del suelo } \\
\hline Indicador & Objetivo & Resultados & Unidad \\
\hline $\begin{array}{l}\text { Vocación Funcional } \\
\text { (Vf) }\end{array}$ & $\begin{array}{l}\text { Conocer el \% real de } \\
\text { ocupación del suelo } \\
\text { según la vocación. }\end{array}$ & $\begin{array}{l}\% \quad \text { vocación } \\
\text { funcional. }\end{array}$ & $\%$ \\
\hline \multicolumn{4}{|c|}{ Atributo: movilidad urbana } \\
\hline Indicador & Objetivo & Resultados & Unidad \\
\hline $\begin{array}{l}\text { Movilidad peatonal } \\
\text { (Mp)/Accesibilidad } \\
\text { al transporte }\end{array}$ & $\begin{array}{l}\text { Reconocer la } \\
\text { importancia del papel } \\
\text { del peatón en favor de }\end{array}$ & $\begin{array}{l}\% \text { de la } \\
\text { superficie para la } \\
\text { movilidad }\end{array}$ & $\% /$ ha \\
\hline
\end{tabular}




\begin{tabular}{|c|c|c|c|}
\hline público y otros usos & la movilidad sostenible. & peatonal. & \\
\hline $\begin{array}{l}\text { Movilidad vehicular. } \\
\text { (Mv) / Accesibilidad } \\
\text { al transporte } \\
\text { público y otros usos }\end{array}$ & 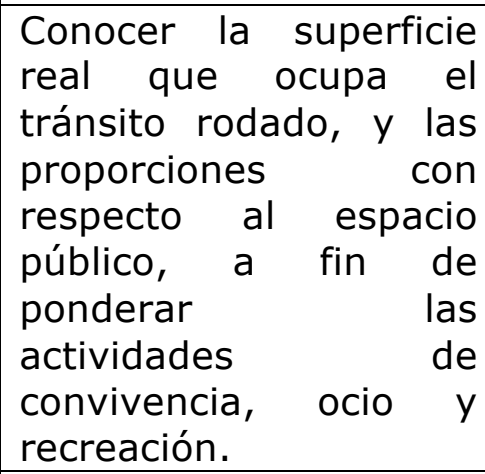 & $\begin{array}{l}\% \text { superficie de } \\
\text { uso vehicular. }\end{array}$ & $\% /$ ha \\
\hline $\begin{array}{l}\text { No de paradas de } \\
\text { transporte público } \\
\text { (Ptp) /Accesibilidad } \\
\text { al transporte } \\
\text { público y otros usos }\end{array}$ & $\begin{array}{l}\text { Garantizar el libre } \\
\text { acceso espacial a la red } \\
\text { de transporte público. }\end{array}$ & $\begin{array}{l}\text { Accesibilidad en } \\
\text { metros lineales } \\
\text { al transporte } \\
\text { público. }\end{array}$ & $\begin{array}{l}\text { No } \\
\text { paradas/ } \\
\text { Min }\end{array}$ \\
\hline \multicolumn{4}{|c|}{ Atributo: espacios públicos } \\
\hline Indicador & \begin{tabular}{|c|} 
Objetivo \\
\end{tabular} & Resultados & Unidad \\
\hline $\begin{array}{l}\text { Superficie de } \\
\text { espacios públicos no } \\
\text { ligados a la } \\
\text { movilidad. (Ep) }\end{array}$ & \begin{tabular}{llr}
\multicolumn{2}{l}{ Conocer el área total } \\
de espacios públicos \\
dedicados & a & la \\
convivencia, & la \\
recreación el ocio y la \\
estancia.
\end{tabular} & $\begin{array}{lr}\% \text { de } & \text { espacios } \\
\text { públicos } & \text { no } \\
\text { ligados a } & \text { la } \\
\text { movilidad. } & \end{array}$ & $\% /$ ha \\
\hline $\begin{array}{l}\text { Accesibilidad a los } \\
\text { espacios públicos. } \\
\text { (Aep }\end{array}$ & $\begin{array}{ll}\text { Establecer } & \text { las } \\
\text { relaciones funcionales y } \\
\text { de accesibilidad de los } \\
\text { ciudadanos a los } \\
\text { espacios públicos. }\end{array}$ & $\begin{array}{l}\text { Distancias / min } \\
\text { para el acceso a } \\
\text { los espacios } \\
\text { públicos. }\end{array}$ & Min/Metros \\
\hline $\begin{array}{l}\text { Espacios públicos de } \\
\text { amortiguación. } \\
\text { (Epa) }\end{array}$ & $\begin{array}{l}\text { Corregir } \\
\text { sobredensificación y la } \\
\text { carencia de m² de } \\
\text { espacios púbicos por } \\
\text { habitante. }\end{array}$ & $\begin{array}{l}\text { Valor de espacios } \\
\text { públicos de } \\
\text { amortiguación. }\end{array}$ & Metros $^{2}$ \\
\hline
\end{tabular}

Fuente: Elaboración propia.

Segunda etapa: Análisis de los resultados. A esta etapa corresponde la generación de los criterios y estrategias de diseño urbano que propendan a la compacidad del sector seleccionado $y$, por ende, a mayores índices de sostenibilidad urbana.

Localización de las áreas problemáticas y de oportunidad
A partir de la realización de los análisis antes descritos, es posible reconocer la factibilidad de la intervención urbana y, por lo tanto, identificar claramente las áreas de oportunidad y aquellas que representarán un reto 


\begin{tabular}{|c|l|}
\hline & $\begin{array}{l}\text { para el desarrollo y la culminación satisfactoria de la } \\
\text { propuesta de diseño urbano. }\end{array}$ \\
\hline Definición de & $\begin{array}{l}\text { Basados en lo anteriormente expuesto, se precisan los } \\
\text { criterios que servirán de base para la intervención de } \\
\text { diseño urbano del sector caso de estudio. }\end{array}$ \\
\hline
\end{tabular}

Tercera etapa: Etapa proyectual. Incluye cuatro instancias:

- Elaboración de la imagen objetivo o plano conceptual.

- Elaboración de la propuesta de diseño urbano.

- Materialización de las estrategias de diseño urbano a nivel de ante proyecto.

- Conclusiones y recomendaciones.

A modo de síntesis se observa en la Figura 2, la estrategia metodológica en la transición de ciudad difusa a ciudad compacta, donde se plasman cada una de las etapas propuestas en esta investigación, comenzando por los componentes conceptuales.

En lo que concierne al modelo de análisis operacional, la revisión del área de estudio comprende, además del diagnóstico conceptual, un diagnóstico tipológico en el que las variables, los indicadores e instrumentos, conllevan a la etapa de síntesis, que culmina con la definición de los criterios de diseño a aplicar en el caso de estudio. Cada uno de los componentes mencionados se desarrolla en cuadros particulares discriminados por atributo. 
REVISTA DE

URBANISMO

ISSN 0717-5051

http://revistaurbanismo.uchile.cl

Revista de Urbanismo N³5 - Diciembre 2016 Departamento de Urbanismo - FAU - Universidad de Chile

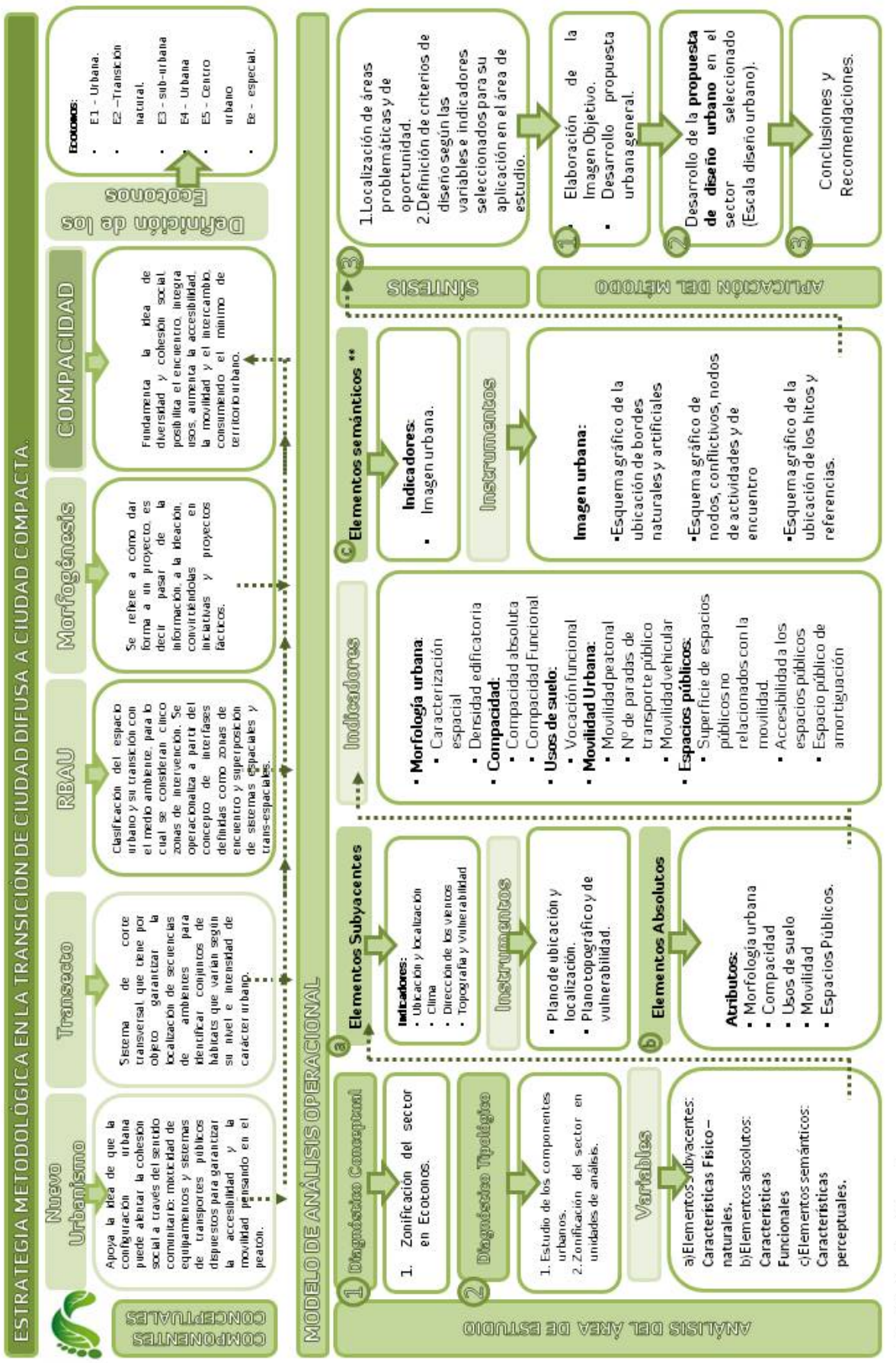

Figura 2. Estrategia metodológica en la transición de ciudad difusa a ciudad compacta.

Fuente: Elaboración propia, 2013. 


\section{Conclusiones}

Esta propuesta, cuyo objetivo principal fue el de proponer un modelo de análisis que incorporara lineamientos tendientes a regular las intervenciones en el ámbito urbano, se desarrolló en dos etapas: la primera, destinada al análisis de postulados que tienen que ver con la visión de ciudad como ecosistema y el diseño urbano ambiental, como vía para lograr la compacidad de las ciudades. La segunda etapa, propositiva, estuvo dirigida a formular un modelo de análisis operacional, que sirviera de base para el establecimiento de criterios de diseño necesarios para la intervención del sector escogido como caso de estudio.

La aplicación de esta estrategia metodológica, es preciso señalarlo, no transgrede normativas generales de ordenación urbana y ambiental vigentes, sino que es susceptible de integrarse dentro de planes macro de ordenación urbanística, local, especial y ambiental.

Finalmente, puede afirmarse que el análisis derivado de la metodología ofrece parámetros susceptibles de guiar la intervención de cualquier zona o de sectores que ofrezcan la interacción de multifocalidades que, al ser tratadas adecuadamente, son susceptibles de conducir a la compacidad urbana y por ende, a su sostenibilidad. Todo ello, sin olvidar que el concepto de ciudad compacta es lo suficientemente amplio como para permitir varias interpretaciones e implementaciones guiadas por la revisión de cualidades y especificidades propias de cada región (diversidad de ambientes complejidad de los procesos urbanos y socioeconómicos, entre otros), y que por ello, para esta investigación se seleccionaron ciertos atributos considerados pertinentes y dejando de lado otros, no aplicables a la realidad latinoamericana. 


\section{Referencias bibliográficas}

Amaya, C. (2011). Ciudad, anticiudad y contraataque: una visión desde la geografía urbana. Pensar a Mérida. Mérida: Universidad de Los Andes (ULA), Academia de Mérida, Centro de Investigación en Gestión Integral de los Riesgos (CIGIR).

Banco Mundial. (2010). Sistemas de ciudades, la urbanización motor del crecimiento y alivio a la pobreza. Estrategia del banco mundial para el sector urbano y los gobiernos locales. Red sobre Desarrollo Sostenible Unidad de coordinación para el sector urbano y los gobiernos locales. Recuperado de http://siteresources.worldbank.org/INTURBANDEVELOPMENT/Resources/3 36387-1269651121606/UrbanStrategy_web_Spanish.pdf

Bartorilla, M. (2009). Articulaciones entre artefacto urbano y ecosistemas naturales: exploraciones proyectuales en los Ecotonos Urbanos de Mérida, Venezuela. RedIALA. Recuperado de http://www.rediala.org/binvestigacion/cuerpo-b resumenes33.html

Camagni, R. Gibelli, M. C, \&, Rigamonti, P. (2002). Urban mobility and urban form: The social and environmental costs of different patterns of urban expansion. Ecological Economics, 40(2), 199-216. https://doi.org/10.1016/s0921-8009(01)00254-3

Carmona, M. (2010). Public places, urban spaces: The dimensions of urban design. Routledge.

Chavoya, J., García, J. y Rendón, H. (2009). Reflexión sobre el modelo urbano: ciudad dispersa, ciudad compacta. Universidad de Guadalajara: México.

Christiaanse, K. (2009). Open city: Designing co-existence. En Network city and landscape. Conferencia realizado en Swiss Federal Institute of Technology (ETH), Zurich.

Duany, A. \& Plater-Zyberk, E. (2003). Transect [página web]. Recuperado de http://www.transect.org

Echenique, M., Hargreaves, A., \& Mitchell, G. (2013). Sustainable cities [clase magistral]. En Internacional Virtual City and Territory: $9^{\circ}$ Congresso Città. Conferencia realizada en Universita degli Studi en Roma, Italia. Recuperado de http://hdl.handle.net/2099/16123

Fumega, J., Niza, S., \& Ferrão, P. (2014). Identification of urban typologies through the use of urban form metrics for urban energy and climate 
change analysis. En Urban Futures-Squaring Circles: Europe, China and the World in 2050. Conferencia realizada en Lisboa, Portugal. http://dx.doi.org/10.13140/2.1.3347.2008

Gaigné, C., Riou, S., \& Thisse, J. F. (2012). Are compact cities environmentally friendly? Journal of Urban Economics, 72(2), 123-136. https://doi.org/10.1016/j.jue.2012.04.001

Girardet, H. (1992). Ciudades alternativas para una vida urbana sostenible. Madrid: Celeste Ediciones, Atlas Gaia.

Manoug, K. (2013). What is mixed use? Presenting an interaction method for measuring land use mix. The Journal of Transport and Land Use, 6(1), 63-72. http://dx.doi.org/10.5198/jtlu.v6i1.291

Pecsi, R. (2002). Desarrollo sostenible en ciudades intermedias: testimonios en América latina. Serie Medio Ambiente y Desarrollo, Cepal, 48, 49-70.

Pesci, R. (2004). ¿Ciudad concentrada o ciudad dispersa? Sustentabilidad y desarrollo urbano. Petrotecnia. Documento No 12, Fundación CEPA, Argentina.

Pergolis, J. (2005). Ciudad fragmentada. Buenos Aires: Editorial Nobuko.

Quintero B., M. (2012). Del medio ambiente al espacio urbano. Ciudades compactas en la transición de ciudades difusas a ciudades compactas. Revista Provincia, 27, enero-junio, 43-76. Recuperado de http://www.redalyc.org/articulo.oa?id=55526545005

Quintero B., M. (2013). Del medio ambiente al espacio urbano: estrategia metodológica en la transición de ciudad difusa a ciudad compacta. Caso de estudio: Av. Alberto Carnevali, Mérida. Mérida: Consejo de Publicaciones de la Universidad de Los Andes.

Remar, P. (2004). El Nuevo Urbanismo, la experiencia de Seaside, Florida. Recuperado https://composicionarqdatos.files.wordpress.com/2008/09/nuevourbanismo_la-experiencia-seaside.pdf

Rueda, S. (1995). Visiones de la ciudad: del urbanismo de Cerda a la ecología urbana. Recuperado de http://www.arkediem.com/ecourbano/imag/ART visiones de la ciudad.pdf.

Rueda, S. (1997). Metabolismo y complejidad del sistema urbano a la luz de la ecología. Recuperado de http://habitat.aq.upm.es/cs/p2/a008.html 
Rueda, S. (2007). La ciudad compacta y diversa frente a la conurbación difusa. Recuperado de http://habitat.aq.upm.es/cs/p2/a009.htm

Salingaros, N. (2007). La ciudad compacta sustituye a la dispersión. Barcelona: Diputació de Barcelona, Colección Territorio y Gobierno, Visiones 6.

Organización de las Naciones Unidas para la Educación, la Ciencia y la Cultura, Unesco. (2011). Programa de reserva de biosfera en ambiente naturales. Recuperado de http://www.unesco.org/new/es/naturalsciences/environment/ecological-sciences/biosphere-reserves/

Westerink, J., Haase, D., Bauer, A., Ravetz, J., Jarrige, F., \& Aalbers, C. B. (2013). Dealing with sustainability trade-offs of the compact city in periurban planning across European city regions. European Planning Studies, 21(4), 473-497. https://doi.org/10.1080/09654313.2012.722927 